\title{
TOWARDS THE DESIGN OF KRESLING TOWER ORIGAMI AS A COMPLIANT BUILDING BLOCK
}

\author{
J. Berre, F. Geiskopf, L. Rubbert, P. Renaud \\ ICube \\ INSA - CNRS - University of Strasbourg \\ john.berre@insa-strasbourg.fr
}

\begin{abstract}
In this paper, the use of the Kresling tower origami as a building block for compliant mechanism design is considered. To help building systems using this origami, models are derived to link the origami pattern geometry to the Kresling tower characteristics. This includes the position of stable configurations, the helical trajectory describing the motion and the orientation of panels during the tower deployment. The provided analytical expressions are helpful to adjust the tower geometry according to desired specification. In addition, an original modification of fold geometry is introduced to modify the tower stiffness. Material removal at specific locations, where maximum fold deformations occur, aims at reducing the actuation force without affecting the kinematics. Experimental evaluation is conducted to assess the relevance of the proposed models and evaluate the impact of fold line modification. The proposed simplified models are precise enough for the synthesis. The capacity to strongly reduce the actuation force, thanks to the the fold line modification, is observed.
\end{abstract}

\section{Introduction}

In the past few years, origami traditional asian art has been increasingly used in several fields of engineering [1, 2]. Indeed, origami has various interesting mechanical properties such as deployability [3], advantageous ratio between stiffness and weight [4], reconfiguration capabilities [5], [6], and for some origami structures, bistability [7]. This has led to different designs of robotic components and systems based on origami [8, 9]. Polymer material is then a relevant choice to keep devices lightweight, using for instance POM [10], PET [8] or PP [9].

Origami structures can be used alone or in combination. In [11], tubular structures based on origami patterns are assembled to form a metamaterial. Each tube can contract along a preferred axis, and has different stiffnesses in other directions. By coupling several tubes in different configurations, a compliant structure is obtained that is deployable in a given direction and stiff in other directions. In [7],

*Address all correspondence to this author. two origamis with different motions are assembled to generate a perilstatic displacement. Another example is in [12], where two origamis with different kinematics are combined to convert a rotational input motion into a translational output motion. For compliant mechanisms, design by association of elementary components is well known with the identification of so-called building blocks, providing specific kinematic properties [13]. This was successfully used in [14] 15]. Origamis belong to the general class of compliant mechanisms. So, identifying origamis that can be used as building blocks, with tools to help their synthesis, should create opportunities to elaborate more complex architectures to be used in robotics.

The Kresling tower origami [16] (Fig. [1) is an interesting structure to go in that direction. It is at the same time a structural element with specific deployment kinematics of its panels, and also a mechanical component with the kinematics of an helical joint that can be used as such for mechanism design. In [17], the origami is used for its deployment capabilities, with the panels used to deploy an antenna. In [8] the Kresling tower is also used for the motion of its panels, the inner radius of the tower being used to guide an endoscope. In [12], the tower is used for its kinematics as the helical motion is used to transform a rotational motion into a translation motion to achieve peristaltic locomotion. Moreover, the Kresling tower can possess two stable configurations. This bistability is in favor of energy efficiency, as no energy is needed to maintain the two stable configurations. It also brings safety, as in absence of actuation, the mechanism remains in a configuration corresponding to one of the two stable states.

In this paper, we consider the Kresling tower origami and bring elements to help the designer building functional components from this structure. Two aspects are covered. The first one concerns the geometrical synthesis of the origami pattern, with the derivation of analytical models describing the deployment of its panels and the overall helical motion. This description of the structure kinematics has not been presented in the literature yet. The second one concerns the structural stiffness of the component, and a way to modify it through a modification at specific locations, where 


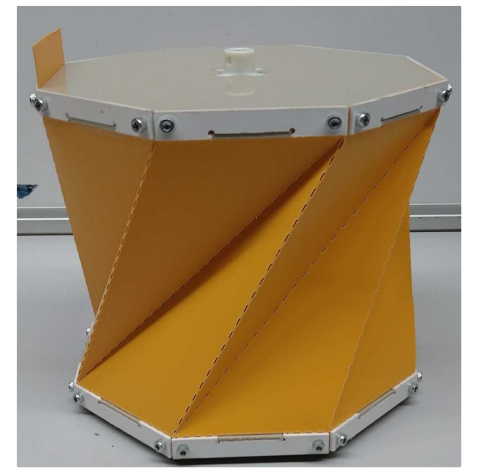

FIG. 1: Prototype of Kresling tower used for assessment

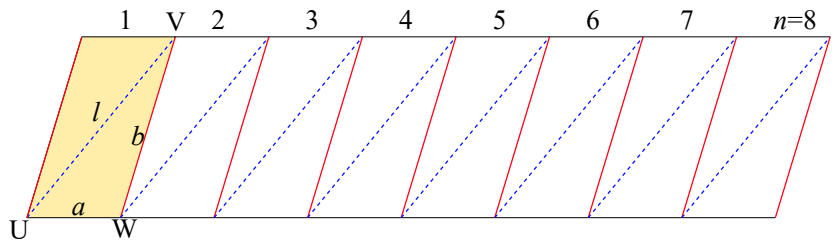

FIG. 2: Pattern of the Kresling tower. Mountain folds depicted with red lines, valley folds by blue dashed lines. The number of elementary patterns is here chosen as $n=8$

fold and panels deformations are observed. One new design parameter is introduced accordingly and its impact is analysed. By providing a set of relationships to help the design of the Kresling tower and an original crease modification to limit stiffness, this paper will help further use of this origami structure in the design of innovative mechanisms.

In section 2] the approach we adopt to model the structure and modify the structure stiffness are introduced and justified. The design characteristics to derive are also selected. The relationships of interest are then developed in section 3 as well as the stiffness modification technique we investigate. Both aspects are assessed experimentally in section 4 Finally, discussion on the results and perspectives in terms of origami design are drawn in Section [5]

\section{Selection of Methods and Design Characteristics \\ 2.1 Desired Characteristics}

The Kresling tower is based on a pattern composed of triangles (Fig. 2). It was named after B. Kresling, because of her work on buckling effect on paper tubes [16], even though similar patterns have been observed earlier in literature [18, 19]. In [20], the tower is built to reach the full folded configuration, i.e. with zero height of the tower, as a first stable configuration. A parameter that controls the triangles definition is used to adjust the energy needed to switch between two stable configurations. Using the completely folded configuration as one stable state is however restrictive from a design point of view. Interestingly, a parameterization proposed in [21] gives the possibility to generate a generalized Kresling tower with a non-flat stable configuration. In this case, the tower has two stable states, that can be desig- nated as low and high configurations. The low configuration corresponds to the state where the tower is compressed, and the high configuration where the tower is deployed as shown on Fig. 3] With this work, the height characterizing these states cannot however be tuned.

The authors in [7] considered the height of the low stable configuration as an input parameter for the synthesis of generalized Kresling towers. In [22], analytical equations, based on the geometry of the tower, allow to determine the pattern dimensions according to the desired stable configurations, characterized by two parameters corresponding to the height of low and high configurations.

To use the Kresling tower as a component in a mechanism, link between the pattern geometry and other characteristics of the tower kinematics is needed. In [8], it is shown the value of the inner radius of the structure, later named $r_{i}$, with the index $i$ used to designate this inner dimension (Fig. 3), is to be evaluated. It is then outlined that the evolution of the inner radius while the height $h$ of the tower (Fig. 3) is varying can be an issue in practical applications. Therefore, modelling the variation of the inner radius during the motion is of importance. The rotation angle $\phi$ between the top and bottom surfaces of the tower is also a key information [7]. In our opinion, the angle between the panel fold lines and the tower axis later, designated as $\rho$, is also to be estimated as it helps describing the panel motions with the outer radius of the structure. In addition, what is needed for design is the variation of these characteristics during the evolution of the tower, from a flat configuration up to a fully extended configuration. To our knowledge, there is no existing model giving analytical expressions of these properties for configurations outside the stable states. In the literature [12, 8, 20], these characteristics are indeed only computed for stable configurations. Our approach is then to derive such a model to access to these characteristics, with explicit relationships between their values and the parameters that define the origami pattern. As a summary, we wish to extract the expressions of $\left(\phi, r_{i}, \rho\right)$ for any tower height $h$. In the following, we will use the index 1 (resp. 2) for the low (high) stable configurations (Fig. 3).

\subsection{Modeling Approach}

Kresling tower origami belongs to the class of nonrigidly foldable origamis [23], which means that the tower cannot be folded without panel deformations. Several works have been introduced to tackle this origami modeling. In [24], the difficulties associated with the use of finite element methods for origami are outlined. Therefore simplified models built by modeling the structure as an assembly of truss are developed. Such a model is of interest as the truss dimensions and arrangement can be linked to the actual origami pattern. The trusses replace the fold lines and their axial deformation is taken into account. The authors introduce virtual folds between the vertices of panels to model the bending stiffness of 4-sided panels, as encountered in some origami tubular designs. For the Kresling tower, 3sided panels have to be modeled thus the method presented 

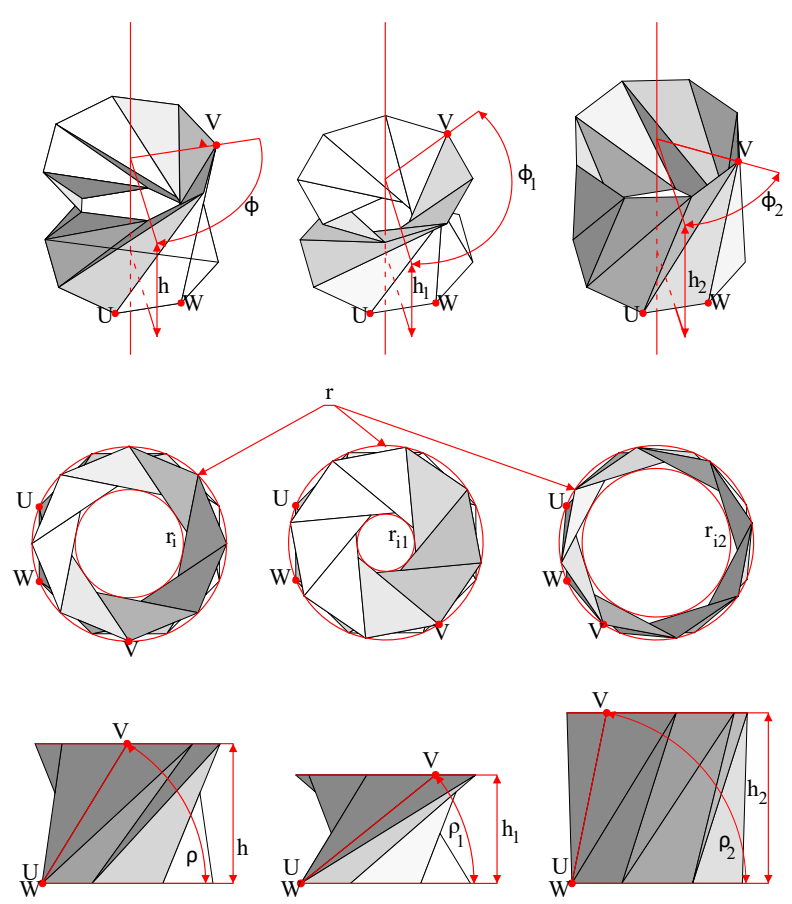

FIG. 3: Representation of the Kresling tower characteristics of interest for a tower height $h$ (left), and for the low (middle) and high (right) stable configurations

in [24] is no more applicable. In [25, 26, 27], a modification of the previous model makes it possible to study the Kresling tower with similar method. However, there is no introduction of virtual folds so the bending stiffness of panels cannot be modeled, whereas it is at the origin of the bistability effect. In [20], a virtual fold line has been added in the Kresling tower pattern to allow rigid foldability. Despite this, the mountain folds of the pattern are deleted at the same time. This seems a rather important simplification, as interactions occurs between the panels in this origami of closed shape. Another limit of the previous works is related to the energy oriented approaches that are used to link the origami deformation to the energy variation during its motion. Energy assessment during origami deployment eases the analysis of bistability, as with other bistable mechanism [28]. However, there is no derived analytical expression that would link the geometry of the tower pattern with the definition of bistable configurations and the aforementioned properties of interest. Our approach is to develop a rigid-bar model based on these previous studies to get such relationships.

\subsection{Stiffness Modulation}

It was shown in [26,24,29] that the origami stiffness can be linked to the stiffness of panels and fold lines. As a consequence, the modulation of an origami stiffness is usually obtained by changing the thickness or material of the origami sheet [30], or by changing the pattern geometry [4]. Thickness variation remains limited, as it impacts the origami kinematics. Material modification can be restricted by the manufacturing techniques and by material requirements due to the application context.

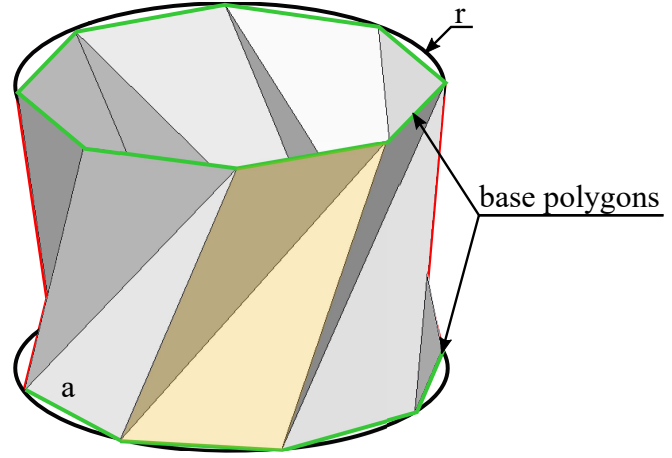

FIG. 4: 3D view of the tower in deployed stable configuration. Top and bottom base polygons are represented in green. Elementary pattern is highlighted in orange and hatched

Another considered fold line modification is a change of geometry all along the fold length. Changing the ratio between strokes and breaks of a dashed line crease pattern is one instance. In [10], this type of approach is studied on a Kresling tower, with a significant reduction of the stiffness of the origami.

In this paper, we propose to investigate a complementary approach to have a strong impact on origami stiffness. It consists in removing material at a specific location, where maximum deformations are encountered along the fold line during the transition between the stable configurations. There are several manufacturing methods to realize a crease, which are dependent on the material or the manufacturing technique of the origami. The double dashed line in [10] is one of them. The approach presented in this paper can be advantageously used in combination with any fold line manufacturing technique. Our objective is in the end to reduce the actuation force required for the tower deployment while respecting the properties adjusted by design of the origami pattern in a first stage.

\section{Models for Design}

The Kresling tower has a kinematic behavior which is equivalent to an helical joint when considering the relative motion of the top and bottom surfaces. In order to describe such kinematics, we focus first on the derivation of the angle $\phi$ as a function of the height $h$. This will be designated as the kinematic model of the Kresling tower. The other characteristics will be derived using the model afterwards. Most of the notations are taken from [27,7] and some equations presented could be defined for stable configurations in [31, 32, 27, 7]. The tower kinematics are dependent on the geometry of the pattern depicted in Fig. 2. We therefore start by presenting the set of parameters used to define the pattern.

\subsection{Tower Parametrization}

The Kresling tower is a closed origami of tubular shape (Fig. 4). The elementary pattern, composed of two triangular panels, is distributed following an axial repetition around the 


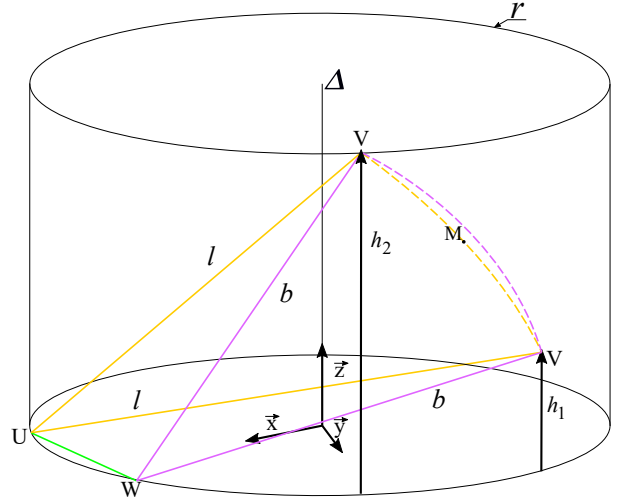

FIG. 5: Representation of the elementary pattern on a 3D view of the tower and motion of bar $l$ and $b$ during deployment.

axis $\Delta$ as shown on Fig. 4. The top and bottom contours are base polygons composed of $n$ sides, with $n$ the number of elementary patterns (Fig. 2). As described in [7], we consider that the base polygons are rigid. The vertices $U, V$ and $W$ (Fig. 5) that define the triangular panels are constrained to move at the same distance from the axis $\Delta$. This is equivalent to constrain the vertices to move on the surface of the cylinder of radius $r$, which circumscribes the base polygons. Because of that, we can restrict the analysis of the Kresling tower motion to the analysis of a single elementary triangle, using cylindrical coordinates.

An elementary pattern is represented in Fig. 2] The parallelogram $U W V W^{\prime}$ is composed of two identical triangles $U V W$ and $U V W^{\prime}$. The lengths $a, b$ and $l$, of respectively segments $U W, V W$ and $U V$, parameterize the geometry of the elementary pattern. The set of parameters $(a, b, l, n, r)$ then defines the geometry of one Kresling tower. The value of length $a$ is linked to $r$ and $n$ (Fig. 6) with $a=2 \cdot r \cdot \sin (\pi / n)$. Thus we define the minimal set of four parameters $\eta=(b, l, n, r)$ to describe the tower geometry.

\subsection{Derivation of Models}

\subsubsection{Kinematic Model Determination}

The kinematic model has to relate the angle $\phi$ to the tower height $h$. This allows us to describe the evolution of the vertex $V$ during the folding, to have then the possibility to reconstruct the panel $U V W$ as in Fig. [5 and finally the whole Kresling tower.

The model derivation is achieved by considering an equivalent rigid-body mechanism. The latter is obtained by replacing the fold lines with bars, connected with spherical joints to the base polygons, also considered as rigid bodies. Without loss of generality, the tower we consider is built from the pattern shown in Fig. 2] to get a left-handed helical shape. There are only two configurations where the mechanism can be assembled. They correspond to the two configurations where the origami tower does not have bending of its panels.

The angle $\phi$ in Fig. [6 is defined as the angle between $\vec{x}$ and the projection of $\overrightarrow{O V}$. The bars $l$ and $b$ have different

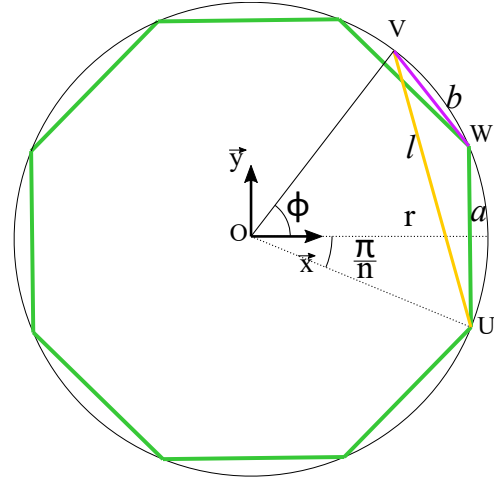

FIG. 6: Projected view of the elementary pattern

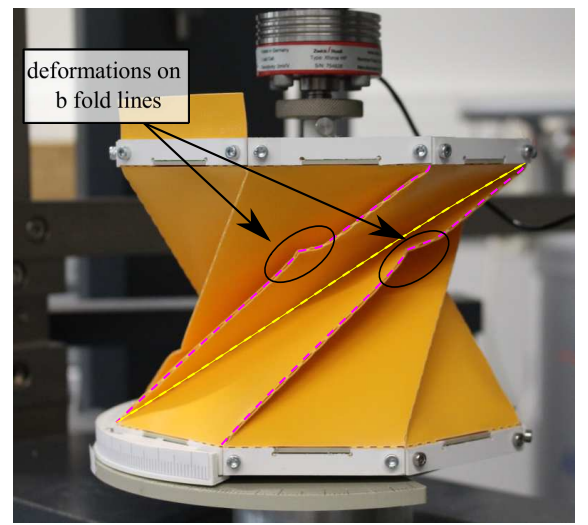

FIG. 7: Observation of fold line deformations during a tower compression

lengths. They are considered rigid and they rotate respectively around $U$ and $W$ (Fig. 5). With this model, the two bars cannot remain connected in $V$ during the origami motion, except for the two stable states. We then define two points $V_{l}$ and $V_{b}$ that are built from the point $V$ but belonging respectively to the bar $l$ and the bar $b$. The points $V_{l}$ and $V_{b}$ are constrained as the other vertices to move at a constant distance $r$ from the axis $\Delta$. This is equivalent in cylindrical coordinates to have a constant radius $r$. The Eqn. (11) links the value of $\phi$ for the points $V_{l}$ and $V_{b}$, respectively $\phi_{l}$ and $\phi_{b}$ to the height $h$ :

$$
\begin{aligned}
& \phi_{l}(h)=2 \cdot \operatorname{asin}\left(\frac{\sqrt{l^{2}-h^{2}}}{2 r}\right)-\frac{\pi}{n} \\
& \phi_{b}(h)=2 \cdot \operatorname{asin}\left(\frac{\sqrt{b^{2}-h^{2}}}{2 r}\right)+\frac{\pi}{n}
\end{aligned}
$$

We have in Eqn. (11) two expressions of the angle $\phi$. Each one corresponds to an hypothesis on the rigidity of the fold line. During the motion between the stable configurations, there are necessarily deformations in the origami structure. We thus need to make an assumption on the deformation of bars during the deployment, to derive the position of the vertex. In [7, 27], the authors faced that same issue and considered the bar $l$ to be rigid, but without giv- 
ing details. We performed various experiments to observe the behavior of the fold lines, corresponding to the bars $l$ and $b$ during origami deployment. We could observe, as illustrated in Fig. 17, that compression along the fold line leads to panel buckling. On the opposite, tension along a fold leads to panel stretching. The stretching of a panel causes negligible deformations compared to a buckling. Thus, we consider for the model the trajectory of the vertex that causes a fold compression.

With Eqn. (1) describing the trajectories of the vertices $V_{i}$ illustrated by Fig. [5, we can deduce that the bar $b$ is solicited in compression in the range of heights $\left[h_{1} h_{2}\right]$ when connected to $V_{l}$. Conversely, the bar $l$ is solicited in traction when connected to $V_{b}$. As a consequence, the trajectory of $V_{l}$ described by $\phi_{l}$ is used to model the motion of the tower between the two stable configurations. On the contrary, outside the interval $\left[h_{1} h_{2}\right]$, the roles are reversed. The trajectory of $V_{b}$ described by $\phi_{b}$ is used consequently to model the motion of the tower outside the range defined by the stable configurations.

Because the bar lengths are constant, our modeling has a given domain of validity. The maximum reachable height corresponds to the length of bars $b$. The value of the minimum reachable height depends on the pattern dimensions. If the bar length $l$ is smaller than the origami diameter, the height $h_{\min }$ is equal to zero. Else, the minimum reachable height can be derived geometrically with $h_{\min }=\sqrt{l^{2}-4 \cdot r^{2}}$. As a consequence, the kinematic model is:

$$
\phi(h)=\left\{\begin{array}{l}
\left.\phi_{b}(h) \text { if } h \in\right] h_{\text {min }} ; h_{1}[\cup] h_{2} ; b[ \\
\phi_{l}(h) \text { if } h \in\left[h_{1} ; h_{2}\right],
\end{array}\right.
$$

\subsubsection{Design Relationships}

With $\eta=(b, l, n, r)$ the set of geometrical parameters that define the pattern geometry, it is possible to derive the heights $\left(h_{1}, h_{2}\right)$ of stable configurations. The stable configuration positions are obtained when $V_{l}=V_{b}$, which can be expressed from the Eqn. (11):

$$
\begin{aligned}
G(h, \eta) & =\phi_{l}(h)-\phi_{b}(h) \\
& =2\left(\operatorname{asin}\left(\frac{\sqrt{l^{2}-h^{2}}}{2 r}\right)-\operatorname{asin}\left(\frac{\sqrt{b^{2}-h^{2}}}{2 r}\right)-\frac{\pi}{n}\right)
\end{aligned}
$$

The designer can then compute the values of heights $h_{1}$ and $h_{2}$ with

$$
\left\{\begin{array}{l}
G\left(h_{1}, \eta\right)=0 \\
G\left(h_{2}, \eta\right)=0
\end{array}\right.
$$

There is a maximum height $H=2 \cdot r \cdot \sqrt{1-\sin \left(\frac{\pi}{n}\right)^{2}}$ of the tower that cannot be exceeded. This height is linked to the radius $r$ and the number of sides $n$. Thus the heights $h_{1}$ and $h_{2}$ must be smaller than $H$, to find a feasible pattern geometry.

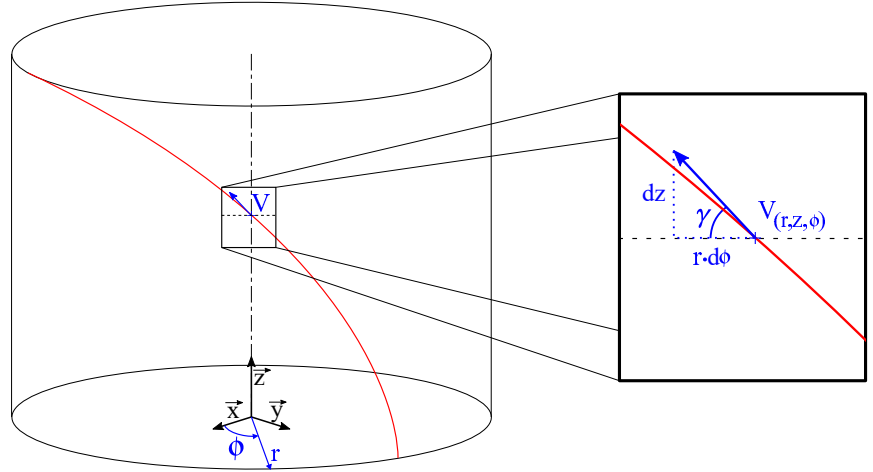

FIG. 8: Definition of the lead angle $\gamma$ : global view with the path of a vertex $V$ in red (left) and close-up in the tangent plane (right)

The inner radius $r_{i}$ is calculated using the projected view along the $\vec{z}$ axis, then calculating the height of the $U O V$ isosceles triangle at $O$. In triangle $U O V$, angle in $O$ is $\phi+\frac{\pi}{n}$ with $\frac{\pi}{n}$ the angle between $(\vec{x}, \overrightarrow{O W})$ and $(\vec{x}, \overrightarrow{O U})$. Using trigonometry, one finally obtains:

$$
r_{i}(h)=\sqrt{r^{2}-4 \cdot r^{2} \sin \left(\frac{\phi(h)+\frac{\pi}{n}}{2}\right)}
$$

The value of the angle $\rho$ between the panels and the plane $(\vec{x}, \vec{y})$ is equal to:

$$
\rho(h)=\operatorname{asin}\left(\frac{h}{l(h) \cdot \sin \left(\frac{l(h)^{2}+a^{2}-b(h)^{2}}{2 \cdot a \cdot b(h)}\right)}\right)
$$

with

$$
\left\{\begin{array}{l}
l(h)=\sqrt{4 \cdot r^{2} \cdot \sin \left(\frac{\phi(h)-\frac{\pi}{n}}{2}\right)} \\
b(h)=\sqrt{4 \cdot r^{2} \cdot \sin \left(\frac{\phi(h)+\frac{\pi}{n}}{2}\right)}
\end{array}\right.
$$

One possible use of the Kresling tower is to build a compliant helical joint. For such type of joint, the designer can be interested by the value of the so-called lead angle, illustrated in Fig. 8 and noted $\gamma$, which describes the relationship between the translational and rotational degrees of freedom. The angle $\gamma(h)$ describes the relationship between the variation of the vertex height $V$ and its angular position. For a given height $h$, it can be computed (Fig. 8) as the ratio between the infinitesimal variation $d h$ and the displacement $r \cdot d \phi$ in the tangential direction, $\gamma(h)=\operatorname{atan}(d h / r . d \phi)$, which becomes 
Table 1: List of tower characteristics with required relationships for their derivation

\begin{tabular}{ccc}
\hline Characteristic & Notation & Eqn. \\
\hline Tower height & $h$ & $(134)$ \\
Tower angle & $\phi$ & (22) \\
Tower inner radius & $r_{i}$ & (5) \\
Angle between panel and horizontal & $\rho$ & (677) \\
Tower helical lead angle evolution & $\gamma$ & $(278)$ \\
\hline
\end{tabular}

$$
\gamma(h)=\left\{\begin{array}{l}
\operatorname{atan}\left(-\frac{\left.\sqrt{l^{2}-h^{2}} \cdot \sqrt{1-\frac{l^{2}-h^{2}}{4 \cdot r^{2}}}\right)}{h}\right) \text { if } h \in\left[h_{1} ; h_{2}\right] \\
\operatorname{atan}\left(-\frac{\sqrt{b^{2}-h^{2}} \cdot \sqrt{1-\frac{b^{2}-h^{2}}{4 \cdot r^{2}}}}{h}\right) \text { otherwise }
\end{array}\right.
$$

The derived relationships are listed in Table 1 with their link to the tower characteristics. These relationships allow the designer to compute the tower characteristics for a given pattern, defined by $\eta$. For a tower synthesis, the way to use the relationships will obviously depend on the design requirements. As there are 4 independent geometrical parameters defining the origami pattern, 4 conditions can be fulfilled simultaneously. As an example, we consider the synthesis of a Kresling tower in section 4 starting from given values of $\left(r, n, h_{1}, h_{2}\right)$, and solve the corresponding system of equations to obtain the parameter set $\eta$.

\subsubsection{Stiffness Modulation}

To modulate the stiffness of the tower, and hence the energy needed for its actuation, we consider a modification of the fold lines. Our proposition is to perform material removal at a specific location along the fold line, to act on the one hand on the stiffness of the fold line, and on the other hand to modify the energy needed for the deformation of the panels adjacent to the fold line. The location of fold modification is identified from experimental observations. The deformations are visible during the folding process such as depicted in Fig. (7) One can see that the deformation of a fold line is not only the expected bending along the line axis. We also note that the deformations are located around the middle of the crease. Consequently, the fold line material removal is being operated at the center of the line as depicted in Fig 9 A prototype of the tower cut with this pattern is visible in Fig. 10. We introduce accordingly a parameter called the "fold density", denoted $F D$, to characterize the modification. It is defined as the ratio between the preserved sections and total length of the fold line. It can vary between $100 \%$, when no modification is made, and $0 \%$. In the following,

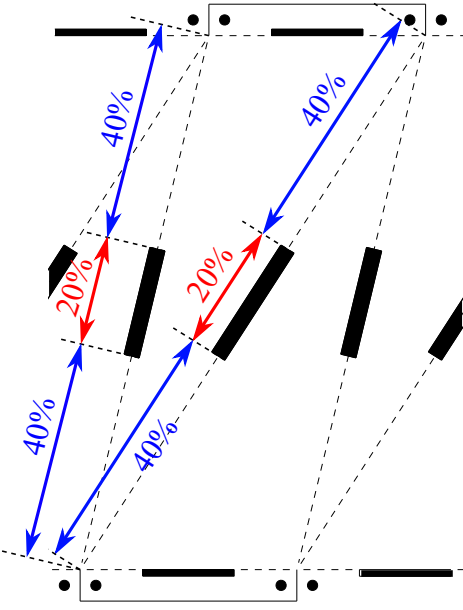

FIG. 9: Example of pattern with $F D=80 \%$. $20 \%$ of fold line represented in black are deleted

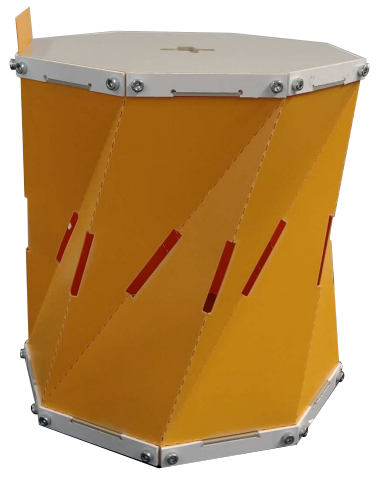

FIG. 10: Prototype of tower $4(F D=80 \%)$

we will assess the impact of this parameter. To do so, the actuation force needed to switch between the two stable configurations can be monitored, as it is related to the structural stiffness. For this evaluation, experimental approach is preferred to numerical simulation, as it will allow us to take into account the material behavior, the possible non linearities introduced by the specific material removal, the manufacturing variabilities, among others.

\section{Fabrication And Experimental Assessment \\ 4.1 Synthesis And Fabrication of Prototypes}

The proposed design approach was applied to build prototypes with different geometries. Here, the goal is to verify the capacity to adjust the position of stable configurations while modifying the behavior of the equivalent helical joint, through modifications of the lead angle value. The design requirements were chosen according to the manufacturing capabilities and the experimental protocol, that are detailed below.

For the origami structure, PP (polypropylene) sheets of $0.5 \mathrm{~mm}$ thickness are used. This material has already been proven for robotic applications for example in [9]. The PP sheets are laser-cut with a Speedy 300 machine from Trotec 
Table 2: Designation and description of design conditions for the prototypes

\begin{tabular}{cccc}
\hline Tower & $h_{1}[\mathrm{~mm}]$ & $h_{2}[\mathrm{~mm}]$ & $\gamma_{\max }\left[^{o}\right]$ \\
\hline 0 & 106.3 & 166.3 & 31.5 \\
1 & 41.5 & 124.7 & 54.3 \\
2 & 80.0 & 130.0 & 39.4 \\
3 & 106.3 & 166.3 & 35.8 \\
\hline
\end{tabular}

Table 3: Designation and description of parameter set $\eta$ for the prototypes

\begin{tabular}{ccccc}
\hline Tower & $b[\mathrm{~mm}]$ & $l[\mathrm{~mm}]$ & $r[\mathrm{~mm}]$ & $n$ \\
\hline 0 & 174.6 & 202.2 & 90 & 8 \\
1 & 139.1 & 174.2 & 90 & 8 \\
2 & 148.5 & 183.5 & 90 & 8 \\
3 & 186.5 & 210.2 & 100 & 12 \\
\hline
\end{tabular}

company. The pattern dimensions are in particular compatible with the laser cutting process and a manual folding.

Tower 0 is considered as a reference tower (Table 2). The value of $h_{2}$ is equal to the maximum reachable height $H$. The height $h_{1}$ is set to $106.3 \mathrm{~mm}$ to reach $60 \mathrm{~mm}$ of stroke between the two stable configurations. Tower 1 is designed to have the stroke between the two stable configurations centered around $H / 2$. Tower 2 is built to reach $50 \mathrm{~mm}$ of stroke with an offset from $H / 2$. For towers 0,1 and 2, the other two conditions for the design were chosen by a direct selection of parameters $r$ and $n$. Finally, tower 3 is built with the same values for $h_{1}$ and $h_{2}$ as for tower 0 , but then adjusting the values of $r$ and $n$ to maximize the lead angle along the deployment of the tower. The corresponding $\eta$ sets for these towers are presented in Table 3 .

A CAD exploded view of prototypes is shown in Fig.113 with designation of the components. The tower is composed of the origami structure which is mounted on rigid ends. The connection is obtained by screwing blocking plates on the periphery of the rigid ends.

As a first step, fold lines are produced without any specific modification. For their manufacturing, 3 processes have been compared, with the shapes depicted in Fig. 11. a dashed line with cutting of minimal width, an intermittent cutting with an oblong profile, and engraving. The minimal cut width is about $0.3 \mathrm{~mm}$ given the laser spot characteristics. Microscopic observations are used to compare the processes. The engraving process ( $\mathrm{C}$ on Fig. 111) leads to fold anisotropy, as the operation is performed on one side only. One engraving step would be needed on each side of the sheet, to alternate the engraving of the mountain and valley

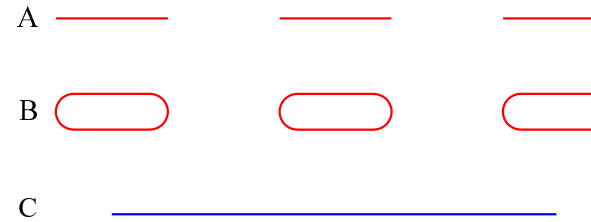

FIG. 11: Sketchs of fold design: patterns of minimal thickness intermittent cutting (A), oblong profile cutting (B) and continuous engraving $(\mathrm{C})$

folds and get isotropic folding. This is time consuming, and positioning errors of the vertices may be introduced. Given the dimensions, the oblong profile (B on Fig. 111) requires a manual separation of the drops, as they stay fused to the polymer sheet after laser machining. This time-consuming phase is avoided by choosing the intermittent cutting, later named dashed line. In addition, the obtained fold lines have an endurance that is comparable to those with the other cutting profiles.

The dashed line pattern is being used with a 1:1 ratio between strokes and breaks, as shown on the cutting pattern in Fig. 12 The dashed lines are positioned to have $5 \mathrm{~mm}$ of material around the vertices, to avoid panel deformation in these regions.

Gluing tabs shown on Fig. 12] were integrated to the origami pattern for closing the shape. The tab size was minimized to limit variation of thickness due to material overlay. The width of the gluing tabs is fixed to $10 \mathrm{~mm}$ to have sufficient contact surface for gluing. Preliminary tests were set up to define the best bonding process. For initial surface treatments, sanding and cleaning, chemical treatment with PPXP, and plasma treatment by Corona effect were compared. Three types of adhesives were considered : neoprene, MS Crystal and cyanoaocrylate. To test the bonding, we used specimens which geometry corresponded to the surface of the tabs used for the tower. The specimens were tested in tension and shear. Panel gluing should not modify strongly the panel stiffness, as it will affect the symmetry of the structure. Bending stiffness was then also assessed. Finally, the most appropriate process is a combination of surface preparation by sanding, cleaning of the gluing area, plasma treatment, and finally use of a neoprene glue.

The rigid ends are obtained by milling of $10 \mathrm{~mm}$-thick PP. Blocking tabs with rectangular holes on the pattern, are added for positioning the tower. The rectangular hole fits in a milled volume on rigid ends. The blocking plates on Fig. 13 applies pressure along the tower, to maintain it in position during the folding process.

\subsection{Validation of Kinematic Behavior}

Before measurements, the towers are submitted to a sequence of 10 foldings and unfoldings using a tensile testing machine, followed by a 30-minute resting period. Loading history is then the same for all prototypes. The number of cycles is chosen based on our observations of a stabilization of the tower behavior. Tower assessment is achieved after this phase. 


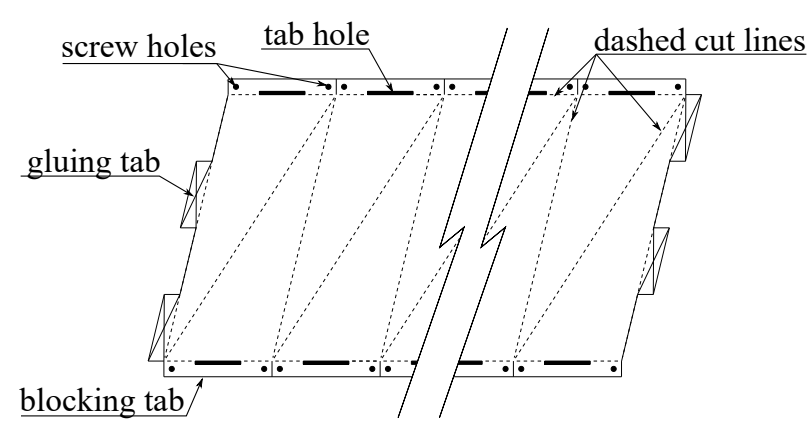

FIG. 12: Cutting pattern for prototypes

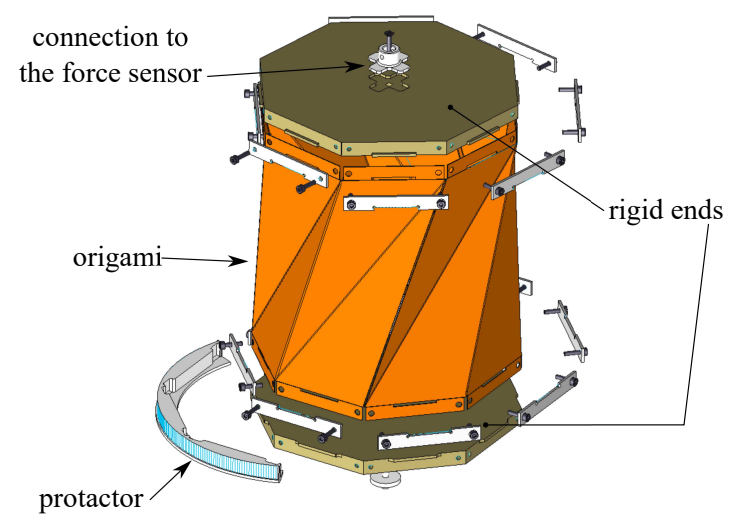

FIG. 13: CAD exploded view of the prototype of tower

Table 4: Measurements of tower heights (standard deviations indicated in brackets) and relative error when compared to the tower radius. The index $m$ stand for measured to differentiate the theoretical value

\begin{tabular}{ccccc}
\hline Tower & $h_{1 m}[\mathrm{~mm}]$ & Error & $h_{2 m}[\mathrm{~mm}]$ & Error \\
\hline 0 & $105.5(0.4)$ & $0.8 \%$ & $165.8(0.1)$ & $0.6 \%$ \\
1 & $51.7(5.0)$ & $11.3 \%$ & $124.6(0.3)$ & $0.1 \%$ \\
2 & $82.5(0.4)$ & $2.7 \%$ & $131.9(0.2)$ & $2.1 \%$ \\
3 & $112.7(1.2)$ & $6.4 \%$ & $165.3(0.1)$ & $1 \%$ \\
\hline
\end{tabular}

the average difference at the position $h_{1}$ is about $5.0 \mathrm{~mm}$, whereas at $h_{2}$, it is about $0.9 \mathrm{~mm}$. We also note that the biggest difference is at the position $h_{1}$ of tower 1 which has the smallest value of $h_{1}$ among the 4 prototypes. The mean relative error on values of Table 4 is about 3\% . Even though refinement of the model might be needed to predict small values of the tower height, the accuracy seems satisfactory, given the simplicity of the model, manufacturing defects and the impacts of PP material non-linearities.

Second, a compression test was performed using a tensile testing machine (Zwick/Roell, Z005) as seen on the Fig. 14. The bottom end is linked to the machine with a cylindrical joint, and the top end is connected to the moving part of the machine, that is linked with prismatic joint to the machine frame. Displacement of the top end is obtained using the integrated sensor. A camera (Canon EOS 700D) is placed in front of the tower, to measure the rotation during the compression test. The camera is used to monitor the value provided by protractor, as shown in Fig. 13. The measuring tool is integrated to the bottom end of the structure, which rotates during the folding. It is then possible to get the time needed to achieve each 1-degree rotation. The translation speed is set by the testing machine, so we are able to compute the corresponding translation carried out by the tower after an initial registration. Finally, the evolution of the tower angle $\phi$ can be plotted as a function of the relative displacement between the tower ends.

In order to validate our model, we plot the angle $\phi$, according to the height $h$ from the Eqn. 2. and compare with the experimental data in black on Fig. 15. On this figure, the yellow curve is the theoretical curve for $\phi_{l}(h)$ and the pink one is for $\phi_{b}(h) . \phi(h)$ curve is represented in solid line from $\phi_{l}$, respectively $\phi_{b}$, according to the definition interval presented in Eqn. 2] We note that the distance, between the experimental curve and the theoretical curve of $\phi(h)$ in solid line, is always smaller than with the dashed lines. This observation confirms our proposition for the expression of the $\phi$ angle outside the stable configurations.

The mean relative error, computed between the experimental evaluation and the values issued from the proposed models, is in the order of $1.5 \%, 0.2 \%, 0.9 \%$ and $0.6 \%$ respectively for towers $0,1,2$ and 3 . These results, below $2 \%$ of error, allow us to validate our model to describe the motion of the tower.

\subsection{Validation of Stiffness Modification}

For this validation, the same experimental protocol is used in terms of initial loading of the prototypes. The compression force is now measured using a force cell (Zwick/Roell Xforce HP 500N), as seen in Fig. 14 The prototypes used to assess the impact of fold density modification are based on the same geometrical set $\eta: b=174.6 \mathrm{~mm}$, $l=202.2 \mathrm{~mm} r=90 \mathrm{~mm}$ and $n=8$. It defines the tower 0 , for which $F D=100 \%$. Towers 4 and 5 are prototypes produced respectively with $F D$ set to $80 \%$ and $60 \%$.

To assess the impact of the proposed fold modification, tower 6 is produced with an homogeneous modification of 


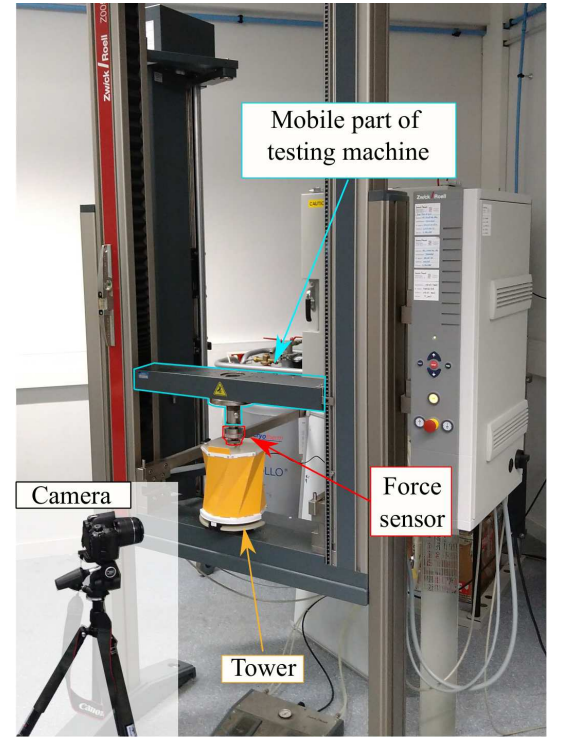

FIG. 14: Prototype during testing with vision-based measurement of rotation

the fold line, where the ratio between strokes and breaks during the laser machining of the fold line has been modified. This tower is characterized by the same amount of material along fold line as the tower 5 with $F D$ set at $60 \%$, but with a homogeneous material distribution along the fold line. This tower is designated by the index $60-h$. Tower 6 is then compared with both tower 0 , which also has a homogeneous material distribution along fold line, and tower 5 , which has the same amount of material along fold line to highlight the relevance of material removing at a specific location.

The maximum compression force $F_{\max }$ is obtained for a compression of about $8 \mathrm{~mm}$ as shown in Fig. 16, The values for towers 0 and 6 are close (Table 5). Reducing the effective length of material along fold lines from $F D=100 \%$ for tower 0 to $60 \%$ for tower 6 causes a reduction of the maximal force by $3.8 \%$. In comparison, the maximal force is lowered by $63 \%$ when using the tower 5 , based on the same amount of material along the fold line than the tower 6 . The impact of the proposed fold line modification is then significantly higher than the one created by a global modification of fold line pattern.

The value of $F_{\max }$ for tower $4(F D=80 \%)$ is reduced by $56 \%$ in comparison with the tower 0 . That means removing $20 \%$ of the fold line leads to a decrease of the maximal force of more than half. The impact of the $F D$ parameter is very significant.

Direct measurements of the height for the stable configurations were also performed. All the towers from Table 5 use the same set of parameters $\eta$, thus the theoretical values of $h_{1}$ and $h_{2}$ are identical and equal to $106.3 \mathrm{~mm}$ and $166.3 \mathrm{~mm}$. The average position difference with theoretical values for towers 0 and 6 is about $0.5 \mathrm{~mm}$, and about $3.6 \mathrm{~mm}$ for towers 4 and 5. For these towers, the mains differences are related to the values of $h_{1}$, which describes the configuration where the tower is compressed at most. The impact
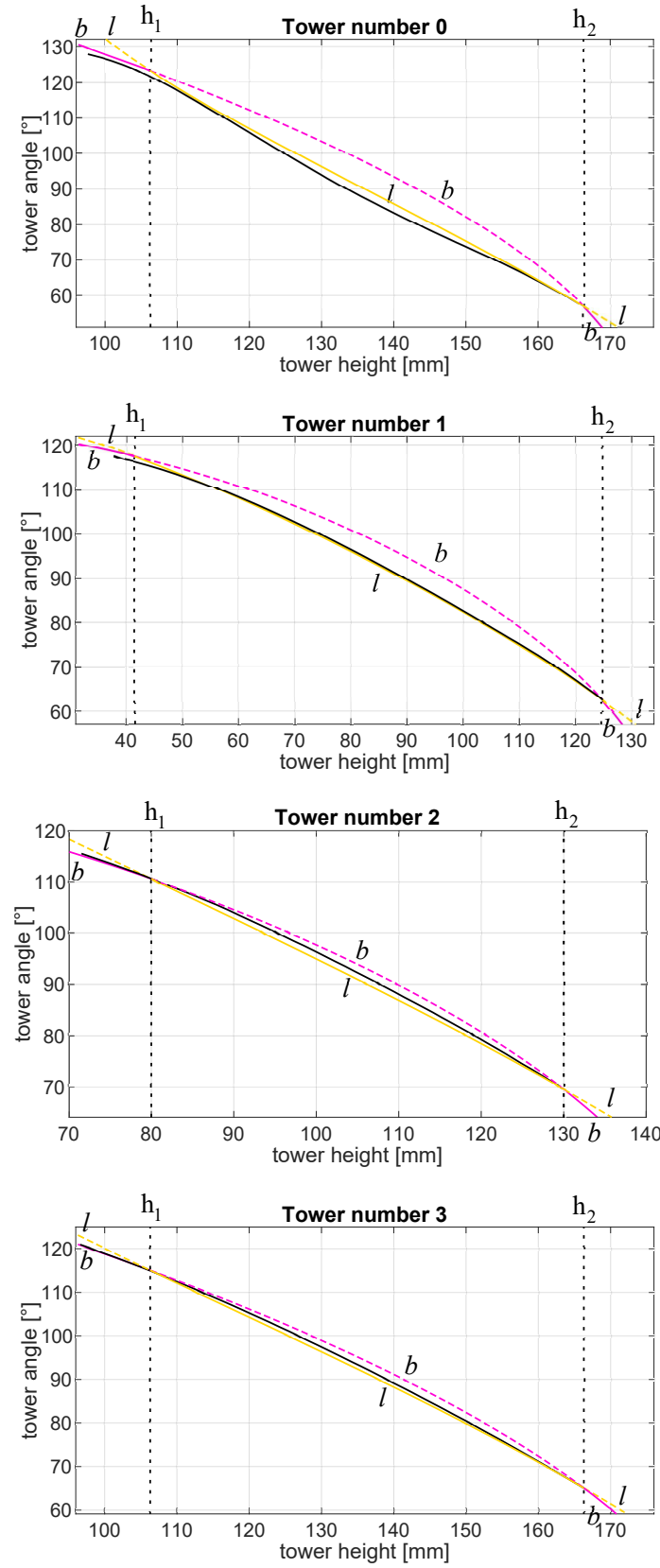

FIG. 15: Tower angle $\phi$ as a function of the tower height $h$. In pink, the theoretical curve for $\phi_{b}$, in yellow, the theoretical curve for $\phi_{l}$ and in black, the measured curve. The solid line compose the curve for theoretical $\phi(h)$ according to the definition interval defined in Eqn. (2)

of fold line modification can be seen as acceptable, as it remains in the order of $5 \%$, even though that will depend on exact application requirements. This means the synthesis of the tower could be achieved in two phases. First the pattern geometry can be defined to achieve the two stable configurations. Then the stiffness level can be modified using the fold modification approach. 


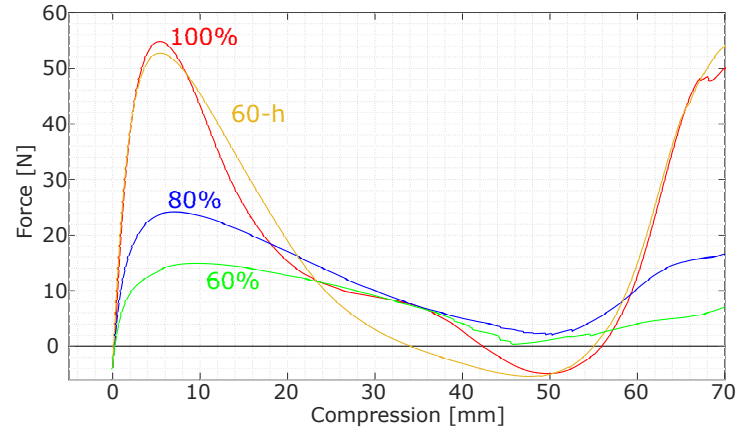

FIG. 16: Force-displacement curves obtained from compression tests of towers described in Table 5

Table 5: Measured values of $h_{1}, h_{2}$ and maximum force for prototypes with modified fold lines

\begin{tabular}{ccccc}
\hline Tower & FD [\%] & $F_{\max }[\mathrm{N}]$ & $h_{1 m}[\mathrm{~mm}]$ & $h_{2 m}[\mathrm{~mm}]$ \\
\hline 0 & 100 & 54.8 & 105.5 & 165.8 \\
4 & 80 & 24.2 & 114.8 & 167.1 \\
5 & 60 & 14.9 & 110.3 & 167.3 \\
6 & $60-\mathrm{h}$ & 52.7 & 105.6 & 166.4 \\
\hline
\end{tabular}

\section{Conclusion}

In this paper, we provided tools to the designer to build compliant components based on the Kresling tower for robotic applications. The origami provides specific motion of its panels, with an overall behavior that can be applied as a bistable helical joint. The tower is then of great interest if associated to a building block design approach. Relationships have been established between the origami pattern and the characteristics of interest which were defined as the position of bistable configurations, the inner radius of the structure, and the orientation of the panels. The experimental results on origami, achieved using materials and processes for suitable with robotic applications, have confirmed the validity of the proposed model.Additionally, an original modification of fold lines at specific locations was introduced to modulate the stiffness of the Kresling tower. Experimental observations confirmed the provided models can be used to select an origami pattern, according to design input parameters. The impact of our specific fold line modification was investigated experimentally as well. Results are confirming the significant impact on stiffness, while the error on the position of stable configurations is not significantly increased by fold modification. This means it seems possible to correct the origami stiffness independently from its kinematics.

Two approaches can be envisioned to be capable of tuning the tower stiffness. The first one would be purely experimental, using a trial and error process. The other one would be based on a model, that can be established using finite element simulation and identification. Model building and comparison of the two approaches constitute the main perspectives of the work.

\section{Acknowledgements}

The authors would like to thank Damien Cartier-Millon, for the definition of the bonding protocol. This work was supported by the French National Agency for Research (ORIGABOT ANR-18-CE33-0008), and Investissements d'Avenir program (Robotex ANR-10EQPX-44).

\section{References}

[1] Greenberg, H., Gong, M., Magleby, S., and Howell, L., 2011. "Identifying links between origami and compliant mechanisms". Mechanical Sciences, 2(2), pp. 217225.

[2] Liu, T., Wang, Y., and Lee, K., 2017. "Threedimensional printable origami twisted tower: Design, fabrication, and robot embodiment". IEEE Robotics and Automation Letters, 3(1), pp. 116-123.

[3] Zirbel, S. A., Lang, R. J., Thomson, M. W., Sigel, D. A., Walkemeyer, P. E., Trease, B. P., Magleby, S. P., and Howell, L. L., 2013. "Accommodating thickness in origami-based deployable arrays". Journal of Mechanical Design, 135(11).

[4] Onal, C. D., Tolley, M. T., Wood, R. J., and Rus, D., 2014. "Origami-inspired printed robots". IEEE/ASME transactions on mechatronics, 20(5), pp. 2214-2221.

[5] Belke, C. H., 2020. From modular origami robots to polygon-based modular systems: a new paradigm in reconfigurable robotics. Tech. rep., EPFL.

[6] Berre, J., Geiskopf, F., Rubbert, L., and Renaud, P., 2021. "Origami-inspired design of a deployable wheel". In New Advances in Mechanisms, Mechanical Transmissions and Robotics, E.-C. Lovasz, I. Maniu, I. Doroftei, M. Ivanescu, and C.-M. Gruescu, eds., Springer International Publishing, pp. 114-126.

[7] Bhovad, P., and Li, S., 2018. "Using Multi-Stable Origami Mechanism for Peristaltic Gait Generation: A Case Study". In International Design Engineering Technical Conferences and Computers and Information in Engineering Conference, Vol. 5B: 42nd Mechanisms and Robotics Conference. V05BT07A061.

[8] Sargent, B., Butler, J., Seymour, K., Bailey, D., Jensen, B., Magleby, S., and Howell, L., 2020. "An origamibased medical support system to mitigate flexible shaft buckling". Journal of Mechanisms and Robotics, 12(4), p. 041005.

[9] Wu, S., Ze, Q., Dai, J., Udipi, N., Paulino, G. H., and Zhao, R., 2021. "Stretchable origami robotic arm with omnidirectional bending and twisting". Proceedings of the National Academy of Sciences, 118(36).

[10] Hwang, H.-Y., 2021. "Effects of perforated crease line design on mechanical behaviors of origami structures". International Journal of Solids and Structures, 230231, p. 111158.

[11] Filipov, E. T., Tachi, T., and Paulino, G. H., 2015. "Origami tubes assembled into stiff, yet reconfigurable structures and metamaterials". Proceedings of the National Academy of Sciences, 112(40), pp. 1232112326. 
[12] Angatkina, O., Chien, B., Pagano, A., Yan, T., Alleyne, A., Tawfick, S., and Wissa, A., 2017. "A metameric crawling robot enabled by origami and smart materials". In Smart Materials, Adaptive Structures and Intelligent Systems, Vol. 58257, American Society of Mechanical Engineers, p. V001T06A008.

[13] Moon, Y.-M., Trease, B. P., and Kota, S., 2002. "Design of large-displacement compliant joints". Journal of Mechanical Design, 36533, pp. 65-76.

[14] Rubbert, L., Bitterli, R., Ferrier, N., Fifanski, S., Vardi, I., and Henein, S., 2016. "Isotropic springs based on parallel flexure stages". Precision Engineering, 43, pp. 132-145.

[15] Merriam, E. G., 2016. Stiffness Reduction Strategies for Additively Manufactured Compliant Mechanisms. Brigham Young University.

[16] Kresling, B., 2008. "Natural twist buckling in shells: from the hawkmoth's bellows to the deployable Kresling-pattern and cylindrical Miura-ori”. In Proceedings of the 6th International Conference on Computation of Shell and Spatial Structures, IASS-IACM 2008, pp. 1-4.

[17] Jape, S., Garza, M., Ruff, J., Espinal, F., Sessions, D., Huff, G., Lagoudas, D. C., Hernandez, E. A. P., and Hartl, D. J., 2020. "Self-foldable origami reflector antenna enabled by shape memory polymer actuation". Smart Materials and Structures, 29(11), p. 115011.

[18] Nojima, T., 2002. "Modelling of folding patterns in flat membranes and cylinders by origami". JSME International Journal Series C Mechanical Systems, Machine Elements and Manufacturing, 45(1), pp. 364-370.

[19] Guest, S. D., and Pellegrino, S., 1994. "The Folding of Triangulated Cylinders, Part I: Geometric Considerations". Journal of Applied Mechanics, 61(4), 12, pp. 773-777.

[20] Pagano, A., Yan, T., Chien, B., Wissa, A., and Tawfick, S., 2017. "A crawling robot driven by multistable origami”. Smart Materials and Structures, 26(9), p. 094007.

[21] Zhang, Q., Cai, J., Li, M., and Feng, J., 2018. "Bistable behaviour of a deployable cylinder with kresling pattern". In Proceedings of the 7th International Meeting on Origami in Science, Mathematics and Education (7OSME), Oxford, UK, pp. 4-7.

[22] Lang, R. J., 2017. Twists, tilings, and tessellations: mathematical methods for geometric origami. CRC Press.

[23] Tachi, T., 2010. "Geometric considerations for the design of rigid origami structures". In Proceedings of the International Association for Shell and Spatial Structures (IASS) Symposium, Vol. 12, Elsevier Ltd, pp. 458-460.

[24] Filipov, E., Liu, K., Tachi, T., Schenk, M., and Paulino, G. H., 2017. "Bar and hinge models for scalable analysis of origami". International Journal of Solids and Structures, 124, pp. 26-45.

[25] Zhai, Z., Wang, Y., and Jiang, H., 2018. "Origamiinspired, on-demand deployable and collapsible me- chanical metamaterials with tunable stiffness". Pro- ${ }_{737}$ ceedings of the National Academy of Sciences, 115(9), pp. 2032-2037.

[26] Liu, K., and Paulino, G., 2017. "Nonlinear mechanics of non-rigid origami: an efficient computational approach". Proceedings of the Royal Society A: Mathematical, Physical and Engineering Sciences, 473(2206), p. 20170348.

[27] Pagano, A., Leung, B., Chien, B., Yan, T., Wissa, A., and Tawfick, S., 2016. "Multi-Stable Origami Structure for Crawling Locomotion". In Smart Materials, Adaptive Structures and Intelligent Systems, Vol. 2: Modeling, Simulation and Control; BioInspired Smart Materials and Systems; Energy Harvesting, p. V002T06A005.

[28] Qiu, J., Lang, J. H., and Slocum, A. H., 2004. "A curved-beam bistable mechanism". Journal of microelectromechanical systems, 13(2), pp. 137-146.

[29] Nayakanti, N., 2016. "Flexigami: folded polygonal unit cells for deployable metamaterials and mechanisms". PhD thesis, Massachusetts Institute of Technology.

[30] Yellowhorse, A., and Howell, L. L., 2018. "Three approaches for managing stiffness in origami-inspired mechanisms". In International Design Engineering Technical Conferences and Computers and Information in Engineering Conference, Vol. 51814, American Society of Mechanical Engineers, p. V05BT07A056.

[31] Jianguo, C., Xiaowei, D., Ya, Z., Jian, F., and Yongming, T., 2015. "Bistable Behavior of the Cylindrical Origami Structure With Kresling Pattern”. Journal of Mechanical Design, 137(6), 06. 061406.

[32] Butler, J., Morgan, J., Pehrson, N., Tolman, K., Bateman, T., Magleby, S. P., and Howell, L. L., 2016. "Highly compressible origami bellows for harsh environments". In Proceedings of the ASME 2016 International Design Engineering Technical Conferences and Computers and Information in Engineering Conference, Vol. 5B: 40th Mechanisms and Robotics Conference. V05BT07A001. 